\title{
Processo de enfermagem aplicado ao paciente com COVID-19 a partir de uma
}

\section{scoping review}

\author{
Nursing process applied to the patient with COVID-19 from a scoping review \\ Proceso de enfermería aplicado al paciente con COVID-19 de una revisión de alcance
}

Recebido: 07/02/2021 | Revisado: 12/02/2021 | Aceito: 18/02/2021 | Publicado: 28/02/2021

Erik Cristóvão Araújo de Melo ORCID: https://orcid.org/0000-0001-5200-3324 Universidade Federal de Campina Grande, Brasil E-mail: erikcristovao@hotmail.com

Juliana Andreia Fernandes Noronha ORCID: https://orcid.org/0000-0002-2990-7744 Universidade Federal de Campina Grande, Brasil E-mail: juli.noronha@gmail.com

Sheila Milena Pessoa dos Santos ORCID: https://orcid.org/0000-0001-9396-9192 Universidade Federal de Campina Grande, Brasil E-mail: Sheila.milena@gmail.com

João Henrique Barbosa Neto ORCID: https://orcid.org/0000-0002-8794-2775 Universidade Federal de Campina Grande, Brasil E-mail: jhenriquebneto@gmail.com

Malena Aparecida da Silva

ORCID: https://orcid.org/0000-0003-2203-0596 Universidade Federal de Campina Grande, Brasil E-mail: malena_xo@hotmail.com

Tcharlys Lopes de Oliveira ORCID: https://orcid.org/0000-0001-5502-8797 Universidade Federal de Campina Grande, Brasil E-mail: Tcharlys.lopes@hotmail.com

\begin{abstract}
Resumo
Objetivos: Propor o processo de enfermagem para pacientes com infecção pela COVID-19 a partir de evidências disponíveis acerca das características clínicas da doença. Metodologia: Scoping review realizada em abril de 2020 em base de dados eletrônicas. Foram incluídos estudos originais, conduzidos com pacientes infectados com a COVID-19, publicados em português, inglês e espanhol. Os estudos foram selecionados conforme recomendação da Preferred Reporting Items for Systematic Reviews and MetaAnalyses. A amostra foi composta por 17 publicações. Resultados: As manifestações clínicas evidenciadas subsidiaram a elaboração de diagnósticos, resultados e intervenções de enfermagem com foco nos domínios: temperatura corporal, infecção, oxigenação/respiração, cardiovascular e função gastrointestinal. Conclusão: Os sistemas de classificação NANDA Internacional, Classificações dos Resultados de Enfermagem e Classificação das intervenções de Enfermagem permitiram elaborar diagnósticos direcionados para avaliação da progressão dos sinais e sintomas e as intervenções tiveram como alvo o combate às causas dos problemas e o monitoramento do quadro clínico dos infectados.

Palavras-chave: Enfermagem; Processo de enfermagem; Terminologia padronizada em enfermagem; COVID-19; SARS-CoV-2; Infecção por Coronavirus.

Abstract

Objectives: Propose the nursing process to patients infected with COVID-19 based on evidences available about the clinical features of the disease. Methodology: Scoping review performed in april 2020 on electronic databases. Were included original studies conducted with patients infected with the disease, published in portuguese, english and spanish. The studies were selected according with the reccomendation of Preferred Reporting Items for Systematic Reviews and MetaAnalyses. The sample was composed with 17 publications. Results: The detected clinical features allowed the elaboration of nursing diagnostics, results and interventions with focus on the domaines: body temperature, infection, oxigenation/respiration, cardiovascular and gastrointestinal function. Conclusion: The systems of classification NANDA International, Classifications of Nursing Outcomes and Classifications of Nursing Interventions allowed to elaborate diagnostics directed to the evaluation of progression of signs and symptoms and the interventions had as target the combat to the causes of the problems and the monitoring of the clinical conditions of the infected.
\end{abstract}


Keywords: Nursing; Nursing process; Standardized nursing terminology; COVID-19; SARS-CoV-2; Coronavirus infection.

\begin{abstract}
Resumen
Objetivos: Proponer el proceso de enfermería para pacientes con infección por COVID-19 a partir de la evidencia disponible sobre las características clínicas de la enfermedad. Metodología: Scoping Review realizada en abril de 2020 en bases de datos electrónicas. Se incluyeron estudios originales, realizados con pacientes infectados por COVID-19, publicados en portugués, inglés y español. Los estudios se seleccionaron según lo recomendado por él Preferred Reporting Items for Systematic Reviews and MetaAnalyses. La muestra estuvo compuesta por 17 publicaciones. Resultados: Las manifestaciones clínicas evidenciadas apoyaron la elaboración de diagnósticos, resultados e intervenciones de enfermería con foco en los dominios: temperatura corporal, infección, oxigenación respiración, función cardiovascular y gastrointestinal. Conclusión: Los sistemas de clasificación NANDA International, Clasificaciones de Resultados de Enfermería y Clasificación de intervenciones de Enfermería permitieron la elaboración de diagnósticos dirigidos a evaluar la progresión de signos y síntomas y las intervenciones dirigidas a combatir las causas de los problemas y monitorear la condición clínica infectada.
\end{abstract}

Palabras clave: Enfermería; Proceso de enfermería; Terminología de enfermería estandarizada; COVID-19; SARSCoV-2; Infección por coronavirus.

\title{
1. Introdução
}

A Coronavirus Disease 2019 (COVID-19) é uma doença infecciosa viral, causada por um novo coronavírus, chamado de SARS-CoV-2, que surgiu no fim do mês de dezembro de 2019, na cidade de Wuhan, província de Hubei na China. Acreditase que o surto teve origem no mercado de frutos do mar, Huanan Seafood Wholesale Market, e, posteriormente, foi identificado em pacientes hospitalizados e em ambientes familiares (Guan, et al, 2019).

Dada a rápida disseminação, o COVID-19 tornou-se uma emergência de saúde pública de interesse internacional e foi declarada como pandemia pela Organização Mundial de Saúde (OMS). Até meados do mês de maio de 2020, foram 4.618.821 casos confirmados e 311.847 mortes (WHO, 2020).

À medida que a pandemia pelo SARS-CoV-2 avança, os sistemas e os serviços de saúde são pressionados por demandas de cuidados especializados. Na linha de frente desses serviços estão os profissionais de saúde, com destaque para enfermagem, devido a necessidade de assistência ininterrupta desses profissionais. Segundo o relatório sobre a situação mundial da enfermagem da OMS, no mundo, são aproximadamente 27,9 milhões de profissionais de enfermagem, que corresponde a 59\% da força de trabalho da saúde. Isso demonstra que no momento atual da luta contra o COVID-19, há milhões de profissionais de enfermagem prestando cuidados primários, secundários e terciários (WHO, 2020).

Nesse contexto, verifica-se a necessidade de promover a oferta de cuidados de enfermagem, pautada na Prática Baseada em Evidência (PBE), para a assistência de qualidade ao paciente com COVID-19. Portanto, coloca-se como essencial o Processo de Enfermagem (PE), que consiste no método assistencial que oferece suporte para tomada de decisão, com o objetivo de investigar, diagnosticar, planejar, implementar e avaliar as ações de cuidado (Alfaro-Lefevre, 2014).

Para a elaboração do PE, dentre os diversos sistemas de classificação, destaca-se a NANDA Internacional (NANDA-I), com seus fatores relacionados, fatores de risco, condições associadas, população em risco e características definidoras; a Classificação dos Resultados de Enfermagem (NOC), com as as escalas de medidas e os indicadores; e a Classificação das Intervenções de Enfermagem (NIC), com suas atividades. Todas as classificações têm o intuito de padronizar a linguagem dos diagnósticos, resultados e intervenções de enfermagem (Alfaro-Lefevre, 2014; NANDA International, 2018; Moorhead; Johnson; Maas; Swanson, 2016; Bulechek; Butcher; Dochterman, 2016).

Salienta-se que ao considerar a pandemia da COVID-19, bem como a ausência de pesquisas com foco na assistência de enfermagem, este estudo pode proporcionar a identificação dos diagnósticos mais relevantes, resultados e intervenções de enfermagem. Isso favorece a escolha de uma linguagem padronizada a ser utilizada no PE para qualificar a assistência de enfermagem. 
Assim, o objetivo desse estudo é propor o processo de enfermagem para pacientes com infecção pela COVID-19 a partir de evidências disponíveis acerca das características clínicas.

\section{Metodologia}

Trata-se de uma Scoping Review, de caráter descritivo, com abordagem quantitativa (Pereira, 2018). Esta pesquisa foi conduzida por meio de protocolo de revisão sistemática para identificar a produção científica relevante e sintetizar evidências acerca de um tópico (Tricco, et al, 2018). Essa etapa possibilitou a produção de evidências para organização da segunda etapa do estudo que consistiu em elaborar uma proposta do PE (diagnósticos, resultados e intervenções de enfermagem) aplicada ao paciente com COVID-19, baseada no sistema de classificação da NANDA-I, NOC e NIC (NANDA International, 2018; Moorhead; Johnson; Maas; Swanson, 2016; Bulechek; Butcher; Dochterman, 2016).

A realização da Scoping Review atendeu aos seguintes tópicos: identificação da questão de pesquisa, identificação dos estudos relevantes, seleção dos estudos, extração e análise dos dados, síntese e comunicação dos resultados (Arskey; O'malley, 2005).

\section{Identificação da questão de pesquisa}

A elaboração da pergunta de pesquisa foi realizada por meio da estratégia PICO, que representa um acrônimo para Paciente, Intervenção/ diagnóstica, Comparação e "Outcomes" (desfecho). Para tanto, foram considerados: (P) pacientes com confirmação da infecção COVID-19 hospitalizados; (I) Preventiva (fatores de risco e comorbidades), Diagnóstica (sinais e sintomas, exames laboratoriais e de imagem) para identificação dos fatores relacionados e características definidoras do diagnóstico de enfermagem (NANDA-I) e intervenções de enfermagem (NIC); (O) resultados de enfermagem (NOC). Considerando o objetivo e desenho do estudo a comparação (C) não pode ser realizada. Assim, a questão de pesquisa delimitada foi: Quais são as características clínicas que subsidiam os diagnósticos, resultados e intervenções de enfermagem para o paciente com Covid-19?

\section{Identificação e seleção dos estudos relevantes}

Foram incluídos artigos originais, nos idiomas espanhol, inglês, português, publicados no ano de 2020, que trataram das características clínicas de pacientes adultos diagnosticados com COVID-19. Portanto, estudos que não correspondem à temática, conduzidos com populações específicas, como crianças, gestantes e profissionais de saúde foram excluídos. O tipo e a qualidade da evidência foram avaliados (Stetler; Brunell; Giuliano; Morsi; Prince; Newell-Stokes, 1998), sendo excluídos cartas ao editor, correspondências, relatos de caso, editoriais, revisões de literatura e opinião de especialistas.

O levantamento dos dados foi realizado com artigos publicados até 15 de abril de 2020, nas seguintes bases de dados: Medical Literature Analysis and Retrieval System Online (MEDLINE), consultada pela U.S. National Library of Medicine National Institute of Health (PubMed), Literatura Latino-Americana e do Caribe em Ciências da Saúde (LILACS) e Índice Bibliográfico Espanhol de Ciências da Saúde (IBECS), por meio da Biblioteca Virtual da Saúde (BVS). A busca nas bases de dados foi realizada a partir do operador booleano OR e os seguintes descritores: "COVID-19", "SARS-CoV-2" e "Coronavirus". Os descritores classificados foram aqueles controlados e disponíveis pelo Medical Subject Headings (MeSH) e Descritores em Ciências da Saúde (DeCS).

\section{Extração e análise dos dados}

De acordo com os critérios de inclusão e exclusão, dois pesquisadores realizaram a leitura cuidadosa dos títulos e resumos de forma independente. As discordâncias foram resolvidas por consenso entre os pesquisadores. Em seguida, foi 
realizada a síntese qualitativa a partir da leitura integral de todos os artigos selecionados, sendo mais uma vez excluídos, quando necessário (Figura 1).

Figura 1 - Processo de identificação e inclusão dos estudos nas bases de dados, 2020.

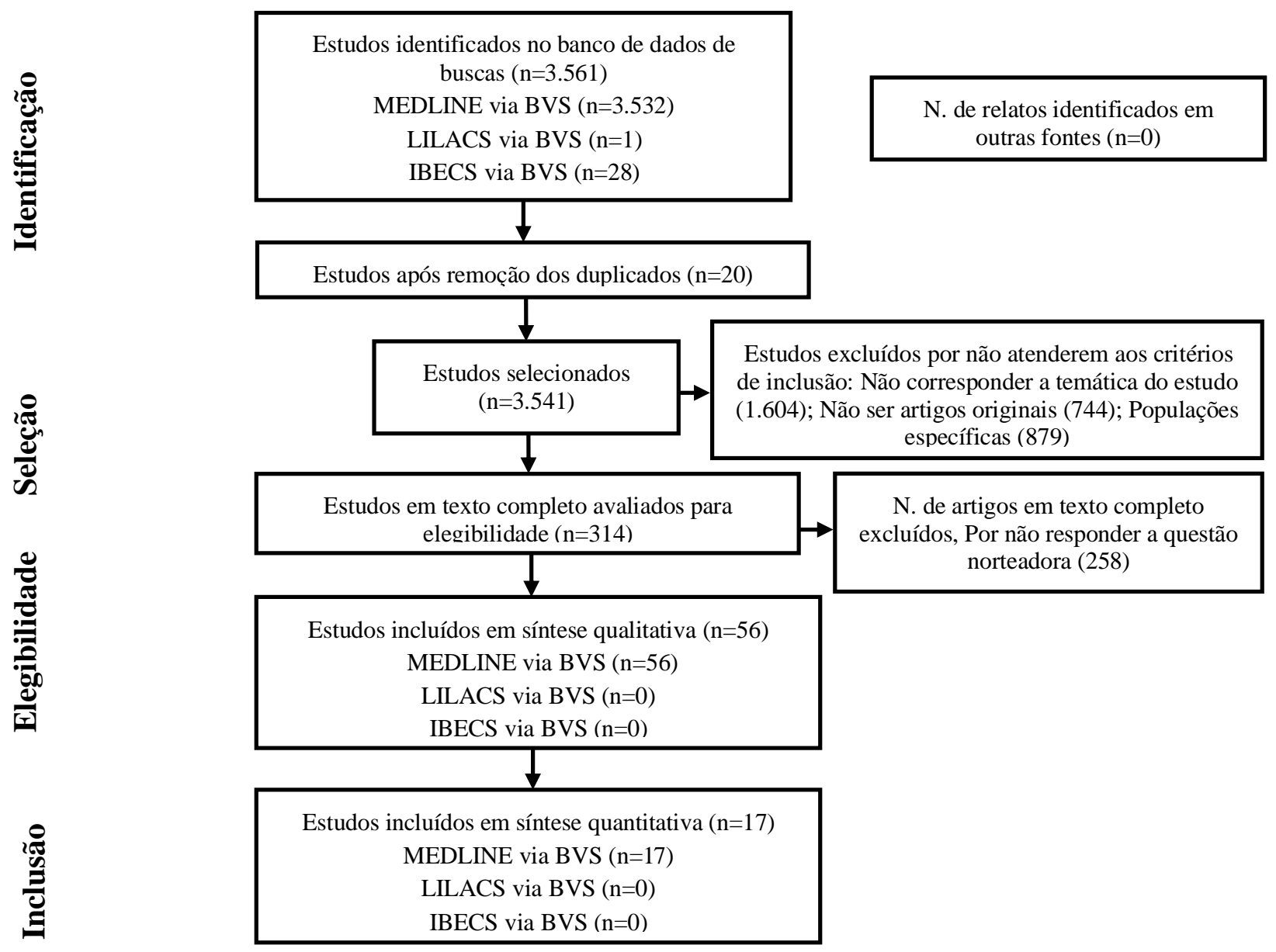

Fonte: Autores.

As evidências acerca das características clínicas dos pacientes foram registradas e organizadas com auxílio do software Excel®. Os resultados foram apresentados por meio de quadros e tabelas, com os dados dos estudos: título, data de publicação, objetivos, desenho do estudo, amostra, fatores de risco, sinais e sintomas e complicações.

\section{Síntese e comunicação dos resultados}

As evidências encontradas na primeira etapa subsidiaram o arcabouço teórico para sugestão dos diagnósticos, resultados e intervenções de enfermagem aplicadas ao paciente diagnosticado com COVID-19. Desse modo, de acordo com a NANDA-I (NANDA International, 2018), os fatores etiológicos e de risco encontrados no estudo foram considerados como fatores relacionados, fatores de risco, condições associadas e população em risco e os sinais e sintomas, por sua vez, foram elencados como características definidoras. Optou-se por priorizar as características clínicas referentes aos sistemas: respiratório, cardiovascular e gastrointestinal, visto que foram as alterações mais encontradas nos artigos da amostra do estudo.

Para os resultados e intervenções utilizamos a Classificação dos Resultados de Enfermagem (Moorhead; Johnson; Maas; Swanson, 2016) e a Classificação das Intervenções de Enfermagem (Bulechek; Butcher; Dochterman, 2016), indicando os seus 
títulos, respectivamente. As ligações entre NANDA-I, NOC e NOC foram utilizadas para potencializar a relação entre os sistemas (Johnson; Moorhead; Bulechek; Butcher; Maas; Swanson, 2012).

\section{Resultados}

Foram incluídos 17 artigos, que totalizam 53.264 mil pacientes com diagnóstico de infecção pela COVID-19. Os estudos foram publicados entre janeiro e abril de 2020, todos conduzidos na China, com os seguintes desenhos: coorte retrospectiva $(29,4 \%)$, caso controle $(5,8 \%)$, série retrospectiva de casos $(41,1 \%)$, descritivo $(5,8 \%)$, revisão sistemática, meta-análise $(5,8 \%)$, estudo observacional retrospectivo (5,8\%), estudo observacional multicêntrico (5,8\%). Os artigos incluídos estão listados e detalhados no Quadro 1.

Quadro 1 - Caracterização dos estudos sobre COVID-19, considerando título, objetivo, tipo de estudo e amostra. Campina Grande, Brasil, 2020.

\begin{tabular}{|c|c|c|c|c|}
\hline $\mathbf{N}^{0}$ & Título & Objetivo do Estudo & Tipo de Estudo & Amostra \\
\hline 1 & $\begin{array}{l}\text { Clinical Characteristics of Coronavirus Disease } \\
2019 \text { in China (GUAN, et al, 2019) }\end{array}$ & $\begin{array}{l}\text { Características clínicas de pacientes infectados } \\
\text { pelo COVID- } 19 \text {. }\end{array}$ & $\begin{array}{l}\text { Coorte } \\
\text { retrospectiva }\end{array}$ & 1324 \\
\hline 2 & $\begin{array}{l}\text { Clinical course and risk factors for mortality of } \\
\text { adult inpatients with COVID-19 in Wuhan, China: a } \\
\text { retrospective cohort study (ZHOU, et al, 2020) }\end{array}$ & $\begin{array}{l}\text { Estabelecer os fatores de risco para } \\
\text { mortalidade e o curso clínico da doença } \\
\text { (incluindo a disseminação viral) }\end{array}$ & $\begin{array}{l}\text { Coorte } \\
\text { retrospectiva }\end{array}$ & 813 \\
\hline 3 & $\begin{array}{l}\text { Clinical characteristics and imaging manifestations } \\
\text { of the } 2019 \text { novel coronavirus disease (COVID- } \\
\text { 19): A multi-center study in Wenzhou city, Zhejiang, } \\
\text { China (YANG, et al, 2020b) }\end{array}$ & $\begin{array}{l}\text { Descrever as características clínicas e } \\
\text { manifestações de imagem de pacientes } \\
\text { hospitalizados com COVID-19. }\end{array}$ & $\begin{array}{l}\text { Coorte } \\
\text { retrospectiva }\end{array}$ & 149 \\
\hline 4 & $\begin{array}{l}\text { Clinical characteristics of } 113 \text { deceased patients } \\
\text { with coronavirus disease 2019: retrospective study } \\
\text { (CHEN, et al, 2020) }\end{array}$ & $\begin{array}{l}\text { Delinear as características clínicas dos } \\
\text { pacientes com doença de coronavírus } 2019 \\
\text { (covid-19) que morreram. }\end{array}$ & $\begin{array}{l}\text { Coorte } \\
\text { retrospectiva }\end{array}$ & 799 \\
\hline 5 & $\begin{array}{l}\text { Clinical characteristics of fatal and recovered cases } \\
\text { of coronavirus disease } 2019 \text { (COVID-19) in Wuhan, } \\
\text { China: a retrospective study (DENG, et al, 2020) }\end{array}$ & $\begin{array}{l}\text { Analisar as características clínicas dos } \\
\text { pacientes que sucumbiram e que se } \\
\text { recuperaram da nova doença de coronavírus de } \\
\text { 2019(COVID-19). }\end{array}$ & Caso controle & 964 \\
\hline 6 & $\begin{array}{l}\text { Clinical characteristics of refractory COVID-19 } \\
\text { pneumonia in Wuhan, China (MO, et al, 2020) }\end{array}$ & $\begin{array}{l}\text { Esclarecer as características de pacientes com } \\
\text { COVID-19 refratário. }\end{array}$ & $\begin{array}{l}\text { Série retrospectiva } \\
\text { de casos }\end{array}$ & 155 \\
\hline 7 & $\begin{array}{l}\text { Clinical Features of } 69 \text { Cases with Coronavirus } \\
\text { Disease } 2019 \text { in Wuhan, China (WANG; YANG; } \\
\text { LI; WEN; ZHANG, 2020) }\end{array}$ & $\begin{array}{l}\text { Descrever as características epidemiológicas, } \\
\text { clínicas, laboratoriais e radiológicas, } \\
\text { tratamento e prognóstico de pacientes com } \\
\text { infecção confirmada por SARS-CoV-2 }\end{array}$ & $\begin{array}{l}\text { Série retrospectiva } \\
\text { de casos }\end{array}$ & 69 \\
\hline 8 & $\begin{array}{l}\text { Clinical characteristics of novel coronavirus cases in } \\
\text { tertiary hospitals in Hubei Province (LIU, et al, } \\
\text { 2020) }\end{array}$ & $\begin{array}{l}\text { Relatar as manifestações clínicas, resultados } \\
\text { dos exames laboratoriais, características de } \\
\text { imagem e regime de tratamento de pacientes } \\
\text { infectados com COVID-19 }\end{array}$ & $\begin{array}{l}\text { Série retrospectiva } \\
\text { de casos }\end{array}$ & 137 \\
\hline 9 & $\begin{array}{l}\text { Clinical Features and Treatment of COVID-19 } \\
\text { Patients in Northeast Chongqing (WAN, et al, 2020) }\end{array}$ & $\begin{array}{l}\text { Descrever os aspectos epidemiológicos, } \\
\text { clínicos, resultados laboratoriais, } \\
\text { características radiológicas, tratamento e } \\
\text { resultados de Pacientes COVID-19 }\end{array}$ & $\begin{array}{l}\text { Série retrospectiva } \\
\text { de casos }\end{array}$ & 135 \\
\hline 10 & $\begin{array}{l}\text { Clinical Features and Short-term Outcomes of } 102 \\
\text { Patients with Coronavirus Disease } 2019 \text { in Wuhan, } \\
\text { China (CAO, et al, 2020) }\end{array}$ & $\begin{array}{l}\text { Investigar os aspectos clínicos e laboratoriais, } \\
\text { características e resultados a curto prazo de } \\
\text { pacientes com COVID- } 19 \text {. }\end{array}$ & $\begin{array}{l}\text { Série retrospectiva } \\
\text { de casos }\end{array}$ & 102 \\
\hline 11 & $\begin{array}{l}\text { Epidemiological and clinical characteristics of } 99 \\
\text { cases of } 2019 \text { novel coronavirus pneumonia in } \\
\text { Wuhan, China: a descriptive study (CHEN, et al, } \\
\text { 2020) }\end{array}$ & $\begin{array}{l}\text { Esclarecer melhor as características } \\
\text { epidemiológicas e clínicas da pneumonia } \\
\text { COVID-19. }\end{array}$ & Estudo descritivo & 99 \\
\hline
\end{tabular}




\begin{tabular}{|c|c|c|c|c|}
\hline 12 & $\begin{array}{l}\text { Hospitalization and Critical Care of } 109 \text { Decedents } \\
\text { with COVID-19 Pneumonia in Wuhan, China (DU, } \\
\text { et al, 2020) }\end{array}$ & $\begin{array}{l}\text { Descrever o processo de hospitalização e } \\
\text { cuidados intensivos dos falecidos com } \\
\text { COVID-19 pneumonia. }\end{array}$ & $\begin{array}{l}\text { Estudo } \\
\text { observacional } \\
\text { multicêntrico }\end{array}$ & 109 \\
\hline 13 & $\begin{array}{l}\text { Clinical characteristics and imaging manifestations } \\
\text { of the } 2019 \text { novel coronavirus disease (COVID- } \\
\text { 19): A multi-center study in Wenzhou city, Zhejiang, } \\
\text { China (YANG, et al, 2020b) }\end{array}$ & $\begin{array}{l}\text { Descrever as características clínicas e } \\
\text { manifestações de imagem de pacientes } \\
\text { hospitalizados com COVID-19 }\end{array}$ & $\begin{array}{l}\text { Coorte } \\
\text { retrospectiva }\end{array}$ & 149 \\
\hline 14 & $\begin{array}{l}\text { Clinical course and outcomes of critically ill } \\
\text { patients with SARS-CoV-2 pneumonia in Wuhan, } \\
\text { China: a single-centered, retrospective, } \\
\text { observational study (YANG, et al, 2020c) }\end{array}$ & $\begin{array}{l}\text { Investigar pacientes críticos, pacientes com } \\
\text { pneumonia confirmada por SARS-CoV-2 e a } \\
\text { morbidade e mortalidade associadas à SARS- } \\
\text { CoV-2. }\end{array}$ & $\begin{array}{l}\text { Estudo } \\
\text { observacional } \\
\text { retrospectivo }\end{array}$ & 52 \\
\hline 15 & $\begin{array}{l}\text { Clinical Characteristics of } 138 \text { Hospitalized Patients } \\
\text { With } 2019 \text { Novel Coronavirus-Infected Pneumonia } \\
\text { in Wuhan, China (WANG, et al, 2020a) }\end{array}$ & $\begin{array}{l}\text { Descrever as características epidemiológicas e } \\
\text { clínicas da Pneumonia do novo coronavírus } \\
(2019 \text { n-CoV). }\end{array}$ & $\begin{array}{l}\text { Série retrospectiva } \\
\text { de casos }\end{array}$ & 138 \\
\hline 16 & $\begin{array}{l}\text { Imaging and clinical features of patients with } 2019 \\
\text { novel coronavirus SARS-CoV-2: A systematic } \\
\text { review and meta-analysis (CAO; LIU; XIONG; } \\
\text { CAI, 2020) }\end{array}$ & $\begin{array}{l}\text { Explorar evidências confiáveis para } \\
\text { diagnóstico e tratamento da COVID-19. }\end{array}$ & $\begin{array}{l}\text { Revisão sistemática } \\
\text { e Meta-análise }\end{array}$ & 46.959 \\
\hline 17 & $\begin{array}{l}\text { COVID-19 in a Designated Infectious Diseases } \\
\text { Hospital Outside Hubei Province, China (CAI, et al, } \\
\text { 2020) }\end{array}$ & $\begin{array}{l}\text { Investigar casos confirmados de COVID-19 } \\
\text { em um hospital }\end{array}$ & $\begin{array}{l}\text { Série retrospectiva } \\
\text { de casos }\end{array}$ & 298 \\
\hline
\end{tabular}

Fonte: Autores.

Dentre as várias características dos pacientes a COVID-19 apresentadas neste estudo, destacaram-se o sexo masculino, idade menor que 60 anos, tendo a doenças malignas, hipertensão e diabetes mellitus como principais comorbidades (Tabela 1).

Tabela 1 - Fatores de risco de pacientes com COVID-19, considerando sexo, idade e doenças comorbidades. Campina Grande, Brasil, 2020.

\begin{tabular}{|c|c|c|c|}
\hline Fatores de risco & Artigos & $\mathbf{n}$ & $\%$ \\
\hline \multicolumn{4}{|l|}{ Sexo } \\
\hline Masculino & $1,2,3,4,5,8,10,11,12,13,14,16,17$ & 13 & 76,4 \\
\hline Feminino & $5,6,7,9$ & 4 & 23,5 \\
\hline \multicolumn{4}{|l|}{ Idade } \\
\hline$<60$ anos & $1,2,8,9,10,11,14,16,17$ & 9 & 52,9 \\
\hline$\geq 60$ anos & $1,2,3,12,17$ & 5 & 29,4 \\
\hline \multicolumn{4}{|l|}{ Comorbidades } \\
\hline Malignidade & $3,4,5,6,7,8,9,10,11,12,13,16,17$ & 14 & 76,4 \\
\hline Hipertensão & $1,3,4,5,6,7,8,9,10,12,14,16,17$ & 13 & 76,4 \\
\hline Diabetes & $1,3,4,5,6,7,8,9,10,12,14,16,17$ & 13 & 76,4 \\
\hline Doença cerebrovascular & $5,6,7,8,9,10,11,12,13$ & 9 & 52,9 \\
\hline Outras doenças cardiovasculares & $1,3,4,9,12,14,16,17$ & 8 & 47 \\
\hline Doença a respiratórias & $3,4,6,9,11,12,13,14$ & 8 & 47 \\
\hline Doença pulmonar obstrutiva crônica & $1,5,6,7,8,10,16$ & 7 & 41,1 \\
\hline
\end{tabular}

Fonte: Autores.

Foram evidenciados no estudo sinais e sintomas leves, moderados e graves, com ênfase para a febre que foi citada em todos os artigos como sintoma principal. Dentre outros sintomas destaca-se no sistema respiratório (tosse e dispneia), sistema 
cardiovascular (taquicardia e palpitações) e gastrointestinais (diarreia, náusea e vômito). De acordo com os exames laboratoriais encontrados, destacaram-se alterações como linfopenia/linfocitopenia, proteína C-reativa alta e dímero D elevado. Em relação aos exames de imagem, destacaram-se a opacidade em vidro fosco e pneumonia bilateral (Tabela 2).

Tabela 2 - Sinais e sintomas, exames laboratoriais e de imagens de pacientes com COVID-19. Campina Grande, Brasil, 2020.

\begin{tabular}{|c|c|c|c|}
\hline Sinais e sintomas & Artigos & $\mathbf{n}$ & $\%$ \\
\hline Febre & $1,2,3,4,5,6,7,8,9,10,11,12,13,14,15,16,17$ & 17 & 100 \\
\hline Tosse & $1,3,4,5,6,7,8,9,10,11,12,13,16,17$ & 14 & 82,3 \\
\hline spneia/ Falta de ar & $1,3,4,5,6,7,8,11,12,13,14,16,17$ & 13 & 76,4 \\
\hline Diarreia & $3,4,6,7,8,10,11,12,13,16,17$ & 11 & 64,7 \\
\hline Expectoração & $1,3,4,6,7,8,12,13,16$ & 9 & 52,9 \\
\hline Náusea e vômito & $3,8,10,11,13,14$ & 6 & 35,2 \\
\hline Taquicardia & $1,3,12$ & 3 & 17,6 \\
\hline Palpitações cardíacas & $4,7,8$ & 3 & 17,6 \\
\hline Dessaturação/SO2\% $<95 \%$ & 8,11 & 2 & 11,7 \\
\hline \multicolumn{4}{|l|}{ Exames Laboratoriais } \\
\hline Linfopenia/linfocitopenia & $1,3,4,5,6,7,8,9,10,11,12,13,14,16,17$ & 15 & 88,2 \\
\hline Proteína c-reativa alta & $3,4,5,6,7,8,10,11,12,13,16,17$ & 12 & 70,5 \\
\hline D dímero alto & $1,3,8,9,12,13$ & 6 & 35,2 \\
\hline Hemoglobina baixa & $1,11,12$ & 3 & 17,6 \\
\hline $\mathrm{PaO} 2$ diminuído & 3,12 & 2 & 11,7 \\
\hline \multicolumn{4}{|l|}{ Exames de Imagens } \\
\hline Opacidade em vidro fosco & $1,6,8,9,10,16$ & 6 & 35,2 \\
\hline Pneumonia bilateral & $1,7,8,11,16$ & 5 & 29,4 \\
\hline Alterações de imagem & $9,10,11,13,14$ & 5 & 29,4 \\
\hline Consolidação pulmonar & $1,6,8$ & 3 & 17,6 \\
\hline Pneumonia unilateral & 11 & 1 & 5,8 \\
\hline
\end{tabular}

Fonte: Autores.

Nos artigos analisados foram mencionadas várias possibilidades de tratamento para os pacientes com COVID-19, destacando-se as intervenções como oxigenoterapia (57,8\%), a ventilação mecânica $(68,4 \%)$, oxigenação por membrana extracorpórea (ECMO) (36,8\%) e cânula nasal de alto fluxo (15,7\%). Com relação ao tratamento medicamentoso, destacou-se a antibioticoterapia e corticoterapia $(73,6 \%)$, a terapia antiviral $(68,4 \%)$ e as imunoglobulinas $(52,6 \%)$.

Foi possível visualizar que as principais complicações dos casos, com destaque para o choque séptico e alterações cardiovasculares (36,8\%), Síndrome do Desconforto Respiratório Agudo (SDRA) (35,5\%) e sepse (15,7\%).

Baseado nas evidências, fatores causais e de risco mais encontrados nos estudos de pacientes com COVID-19, foram elencados e priorizados dez diagnósticos de enfermagem (NANDA-I), resultados e intervenções de enfermagem, baseados na NOC e NIC, respectivamente (Quadro 2). Para melhor direcionamento das relações, foram utilizadas as ligações entre NANDAI, NOC e NIC(11), além da análise das definições, domínios, classes, bem como os indicadores e atividades, respectivos. 
Quadro 2 - Diagnósticos de enfermagem (NANDA-I), Resultados (NOC) e Intervenções (NIC) para pacientes com COVID-19. Campina Grande, Brasil, 2020.

\begin{tabular}{|c|c|c|c|}
\hline Domínio & $\begin{array}{c}\text { Diagnósticos de Enfermagem } \\
\text { (NANDA-I) }\end{array}$ & Resultados (NOC) & Intervenções (NIC) \\
\hline 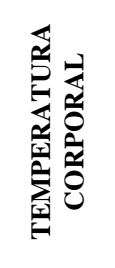 & $\begin{array}{l}\text { Título:(00008) Termorregulação } \\
\text { ineficaz } \\
\text { Fatores relacionados: Doença (COVID- } \\
\text { 19), condição que afeta a regulação da } \\
\text { temperatura, sepse } \\
\text { Características definidoras: aumento } \\
\text { da temperatura corporal acima dos } \\
\text { parâmetros normais, taquicardia }\end{array}$ & $\begin{array}{l}\text { Gravidade da infecção }(0704) ; \\
\text { Resposta ao medicamento }(2301) ; \\
\text { Sinais vitais }(0802) \text {; Termorregulação } \\
(0800)\end{array}$ & $\begin{array}{l}\text { Administração de medicamentos (2308); Controle } \\
\text { hídrico (4120); Monitoração de sinais vitais (6680); } \\
\text { Monitoração hídrica (4130); Regulação da temperatura } \\
\text { (3900); Tratamento da febre (3740) }\end{array}$ \\
\hline \multirow{2}{*}{ 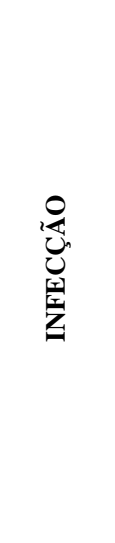 } & $\begin{array}{l}\text { Título:(00205) Risco de choque } \\
\text { Fatores de risco: Infecção (COVID-19), } \\
\text { procalcitonina elevada*, sepse, hipóxia, } \\
\text { hipoxemia }\end{array}$ & 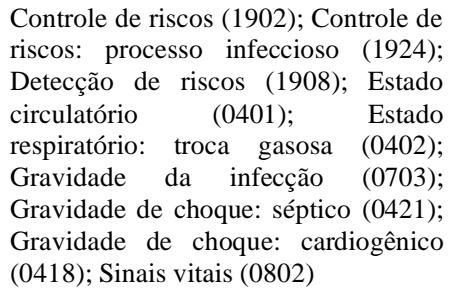 & $\begin{array}{l}\text { Administração de medicamentos (2308); Controle da } \\
\text { hipovolemia (4180); Controle da infecção (6540); } \\
\text { Controle de medicamentos (2380); Cuidados cardíacos } \\
\text { (4040); Identificação de risco (6610); Interpretação de } \\
\text { dados laboratoriais (7690); Monitoração de sinais } \\
\text { vitais (6680); Monitoração respiratória (3350); } \\
\text { Oxigenoterapia (3320); Proteção contra infecção } \\
\text { (6550); Prevenção do choque (4260); Terapia } \\
\text { endovenosa (EV) (4200) }\end{array}$ \\
\hline & 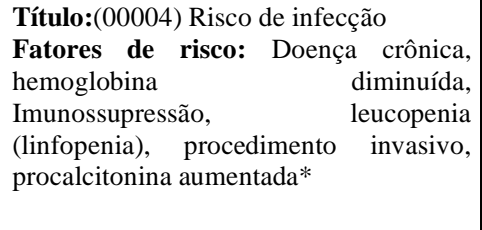 & $\begin{array}{l}\text { Controle de riscos (1902); Controle de } \\
\text { riscos: processo infeccioso (1924); } \\
\text { Detecção do risco (1908); Estado } \\
\text { imunológico (0702); Gravidade da } \\
\text { infecção (0703); Resposta ao } \\
\text { medicamento (2301) }\end{array}$ & $\begin{array}{l}\text { Administração de medicamentos (2308); Controle da } \\
\text { infecção (6540); Controle de doenças transmissíveis } \\
(8820) \text {; Controle de medicamentos (2380); } \\
\text { Identificação de risco (6610); Interpretação de dados } \\
\text { laboratoriais (7690); Monitoração de sinais vitais } \\
\text { (6680); Monitoração respiratória (3350); Proteção } \\
\text { contra infecção (6550) }\end{array}$ \\
\hline 焉 & $\begin{array}{l}\text { Título:(00030) Troca de gases } \\
\text { prejudicada } \\
\text { Fatores relacionados: Alteração da } \\
\text { membrana alveolocapilar (excesso de } \\
\text { muco nos alvéolos) } \\
\text { Características definidoras: Dispneia, } \\
\text { gasometria arterial anormal (paO2), SO2 } \\
\text { baixo, hipoxemia, hipóxia, padrão } \\
\text { respiratório anormal, taquicardia } \\
\text { Título:(00031) Desobstrução ineficaz } \\
\text { das vias aéreas } \\
\text { Fatores relacionados: Muco excessivo, } \\
\text { secreções retidas, exsudado nos alvéolos, } \\
\text { infecção (COVID-19), DPOC } \\
\text { Características definidoras: Alteração } \\
\text { na frequência respiratória (taquipneia, } \\
\text { dispneia, falta de ar), alteração do padrão } \\
\text { respiratório*, escarro em excesso, } \\
\text { alterações nas imagens (TC)* }\end{array}$ & $\begin{array}{l}\text { Controle de riscos: processo infeccioso } \\
\text { (1924); Estado respiratório: } \\
\text { permeabilidade das vias aéreas (0410); } \\
\text { Estado respiratório: troca gasosa } \\
(0402) \text {; Estado respiratório: ventilação } \\
\text { (0403); Gravidade da infecção (0703); } \\
\text { Gravidade dos sintomas (2103); } \\
\text { Hidratação (0602); Resposta à } \\
\text { Ventilação Mecânica: Adulto }(0412) \text {; } \\
\text { Resposta ao desmame da ventilação } \\
\text { mecânica: adulto (0412); Resposta ao } \\
\text { medicamento (2301); Sinais vitais } \\
\text { (0802) }\end{array}$ & $\begin{array}{l}\text { Administração de medicamentos (2308); Aspiração de } \\
\text { Vias Aéreas (3160); Assistência ventilatória (3390); } \\
\text { Controle ácido-básico (1910); Controle da infecção } \\
\text { (6540); Controle da ventilação mecânica: invasiva } \\
\text { (3300); Controle da ventilação mecânica: não invasiva } \\
\text { (3302); Controle de medicamentos (2380); Controle de } \\
\text { Vias Aéreas (3140); Controle hídrico (4120); } \\
\text { Desmame da ventilação mecânica (3310); Estimulação } \\
\text { da Tosse (3250); Fisioterapia Respiratória (3230); } \\
\text { Inserção e Estabilização de Vias Aéreas Artificiais } \\
\text { (3120); Interpretação de dados Laboratoriais (7690); } \\
\text { Monitoração de sinais vitais (6680); Monitoração } \\
\text { hídrica (4130); Monitoração respiratória (3350); } \\
\text { Oxigenoterapia (3320); Posicionamento (0840); } \\
\text { Proteção contra infecção (6550); Terapia endovenosa } \\
\text { (EV) (4200); Testes laboratoriais à beira do leito } \\
\text { (7610) }\end{array}$ \\
\hline \multirow{2}{*}{ 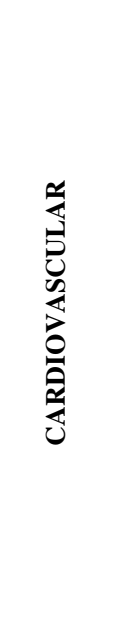 } & $\begin{array}{l}\text { Título:(00200) Risco de perfusão tissular } \\
\text { cardíaca diminuída } \\
\text { Fatores de risco: Diabetes mellitus, } \\
\text { hipertensão, hipoxemia, hipóxia, Proteína } \\
\text { C-reativa elevada } \\
\text { Título:(00240) Risco de débito cardíaco } \\
\text { diminuído } \\
\text { Fatores de risco: Alterações na } \\
\text { contratibilidade, creatina elevada* }\end{array}$ & 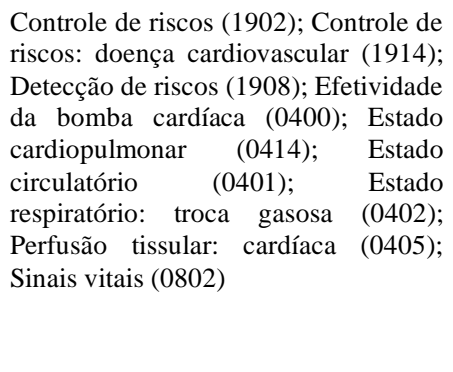 & $\begin{array}{l}\text { Administração de medicamentos (2308); Controle de } \\
\text { arritmias (4090); Controle de choque: cardiogênico } \\
\text { (4254); Controle de medicamentos (2380); Cuidados } \\
\text { cardíacos (4040); Identificação de risco (6610); } \\
\text { Interpretação de dados laboratoriais (7690); } \\
\text { Monitoração hemodinâmica invasiva (4210); } \\
\text { Monitoração de sinais vitais (6680); Monitoração } \\
\text { respiratória (3350); Oxigenoterapia (3320); } \\
\text { Precauções cardíacas (4050); Prevenção do choque } \\
\text { (4260); Regulação hemodinâmica (4150); Terapia } \\
\text { endovenosa (EV) (4200) }\end{array}$ \\
\hline & $\begin{array}{l}\text { Título: }(00268) \text { Risco de } \\
\text { tromboembolismo venoso } \\
\text { Fatores de risco: Admissão em unidade } \\
\text { de terapia intensiva, idade > } 60 \text { anos, } \\
\text { comorbidade médica significativa e } \\
\text { dímero D elevado*. }\end{array}$ & $\begin{array}{l}\text { Conhecimento: prevenção de trombo } \\
\text { (1865); Controle de riscos: trombo } \\
\text { (1932); Controle de riscos (1902); } \\
\text { Detecção de riscos (1908); Estado } \\
\text { circulatório (0401); Perfusão tissular: } \\
\text { periférica (0407) }\end{array}$ & $\begin{array}{l}\text { Administração de medicamentos (2308); Controle de } \\
\text { medicamentos (2380); Controle da sensibilidade } \\
\text { periférica (2660); Controle de terapia trombolítica } \\
\text { (4270); Cuidados circulatórios: insuficiência venosa } \\
\text { (4066); Cuidados na embolia: periférica (4104); } \\
\text { Identificação de risco (6610) }\end{array}$ \\
\hline
\end{tabular}




\begin{tabular}{|c|c|c|c|}
\hline 竞 & 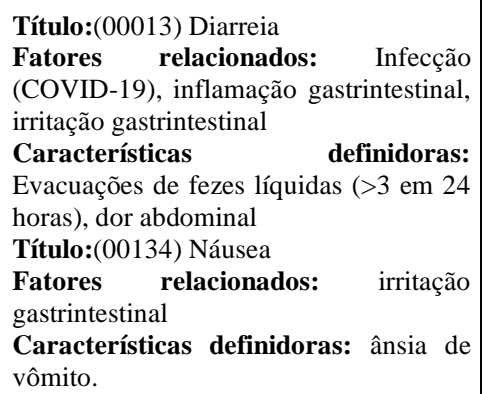 & $\begin{array}{l}\text { Continência intestinal (0500); Controle } \\
\text { da dor (1605); Equilíbrio eletrolítico } \\
\text { (0606); Equilíbrio hídrico (0601); } \\
\text { Eliminação intestinal (0501); Função } \\
\text { gastrointestinal (1015); Hidratação } \\
\text { (0602); Gravidade da infecção (0703); } \\
\text { Resposta ao medicamento (2301) }\end{array}$ & $\begin{array}{l}\text { Administração de medicamentos (2308); Controle da } \\
\text { diarreia (0460); Controle da infecção (6540); Controle } \\
\text { da náusea (1450); Controle da nutrição (1100); } \\
\text { Controle hídrico (4120); Controle intestinal (0430); } \\
\text { Controle de medicamentos (2380); Controle do vômito } \\
\text { (1570); Interpretação de dados laboratoriais (7690) }\end{array}$ \\
\hline
\end{tabular}

*fatores relacionados, fatores de risco e características definidoras que não estão presentes na NANDA-I. Fonte: Autores.

\section{Discussão}

A COVID-19 apresenta-se como grave problema de saúde mundial e é um desafio constante para pacientes, profissionais e gestores de saúde. A identificação dos fatores etiológicos, de risco, sinais e sintomas da doença, fornece ao enfermeiro um arcabouço teórico que fundamenta o PE.

De acordo com os resultados do estudo, constituem-se como fatores de risco para COVID-19 o sexo, a idade e a existência comorbidades. Corroborando esse resultado, metanálise com 10 estudos, realizada com 50.466 pacientes identificou que a maioria (52\%) era do sexo masculino (Sun; Qie; Liu; Ren; Li; Xi, 2020). Essa tendência foi confirmada em análises epidemiológicas conduzidas na Europa e nos Estados Unidos (ECDC, 2002; Garg, et al, 2020). Embora inconclusivos, estudos têm sugerido que a prevalência de comorbidades, o tabagismo, fatores genéticos e os hábitos de vida masculinos possam explicar a predominância nesse grupo (Wenham; Smith; Morgan, 2020).

A distribuição por grupo etário indica que os casos de hospitalização predominaram entre os adultos jovens. Entretanto, estudos que analisaram as hospitalizações demonstraram maior comprometimento causado pela COVID-19 em pessoas mais velhas e com maior número de comorbidades, revelando que esses podem ser fatores de risco críticos para os pacientes (Yang, et al, 2020).

Em nossa análise foram identificadas como principais comorbidades dos pacientes hospitalizados com COVID-19, as doenças hipertensivas, diabetes, doenças cardiovasculares e as doenças pulmonares. Uma metanálise com sete estudos, incluindo 1.576 pacientes sugeriu a prevalência de hipertensão em $21,1 \%$, diabetes, doenças cardiovasculares e doenças dos sistema respiratório em 9,7\%, 8,4\% e 1,5\% dos casos, respectivamente (Yang, et al, 2020a). Os autores do mesmo estudo concluíram que embora seja necessária a realização de estudos clínicos que esclareçam os efeitos da infecção pelo coronavírus em pacientes com comorbidades, sabe-se que as doenças crônicas compartilham processos que favorecem a progressão de distúrbios infecciosos, como o estado pró-inflamatório e a atenuação da resposta imune.

A COVID-19 é uma infecção multifatorial, que leva a sinais e sintomas leves, moderados e graves, afetando vários sistemas, de relevância na morbimortalidade, que pode comprometer o sistema respiratório, cardiovascular e gastrointestinal (Guan, et al, 2020), conforme os fatores de risco e etiológicos e sinais e sintomas apresentados pelo estudo.

Após a entrada do vírus nas células, ocorre uma rápida replicação viral, desencadeando uma resposta imune e inflamatória, que se inicia com uma tempestade de citocinas, principalmente a interleucina 6 (IL-6) que resulta em inflamação local e sistêmica, que pode prejudicar órgãos distantes. A IL-6 é produzida por leucócitos ativados e atua em um grande número de células e tecidos. Essa resposta hiper-inflamatória pode ser confirmada pelo aumento dos níveis de ferritina, interleucinas, procalcitonina e PCR (Joseph, 2020).

Como resposta a este processo infeccioso e inflamatório, foi identificado no estudo como principal alteração nos pacientes, a termorregulação ineficaz. Caracterizada pelo aumento da temperatura corporal, frequência respiratória, e taquicardia. A hipertermia decorre da resposta às condições associadas à doença viral, que atuam sobre o centro termorregulador e 
desencadeiam a produção e conservação de calor (tremores, vasoconstrição periférica e aumento do metabolismo basal (NANDA International, 2018). Essas alterações são mediadas por pirógenos endógenos (citocinas) produzidos em resposta a pirógenos exógenos (vírus) (El-Radhi, 2019).

O resultado esperado das intervenções de enfermagem tem como alvo a manutenção da temperatura corporal dentro de uma variação normal (Moorhead; Johnson; Maas; Swanson, 2016). Para tanto, devem ser realizadas a administração de medicamentos, a monitorização dos sinais vitais e controle hídrico (Bulechek; Butcher; Dochterman, 2016). Salienta-se que o cuidado ao paciente deve ser pautado na avaliação dos sinais clínicos apresentados, inclusive, uso de um método físico para tratamento de febre (Oliveira; Silva; Silva; Chianca, 2016).

Em consonância com nossos resultados, foi identificado em outro estudo que a linfopenia ocorreu em mais de $80 \%$ dos pacientes, sendo o grau dessa alteração um indicador prognóstico importante no início da infecção (Chen, Et Al, 2020; Wang, et $a l, 2020)$. Os mesmos autores desse estudo concluíram que nos pacientes acometidos pela COVID-19, ocorre reduções nos níveis circulantes de linfócitos e uma dominância relativa de células mononucleares (monócitos e macrófagos) nos tecidos das lesões alvo, onde o pulmão foi considerado alvo inicial.

Diante da infecção pelo coronavírus e da resposta inflamatória desencadeada por ele, foram identificados como fatores de risco a presença de doença crônica pré-existente, linfopenia e neutropenia, hemoglobina diminuída e procedimento invasivo para o diagnóstico de enfermagem risco de infecção (NANDA International, 2018). Ressalta-se que o paciente com a COVID19 pode estar sujeito às infecções oportunistas que podem ser virais, bacterianas ou fúngicas, em razão da pneumonia associada à ventilação mecânica ou à infecção hospitalar, que estão associadas a um mau prognóstico (Cascella; Rajnik; Cuomo; Dulebohn; Di Napoli, 2020). Portanto, o diagnóstico de risco direciona as ações de enfermagem para prevenção precoce dessas infecções oportunistas, como sepse, hipoxemia e síndrome da resposta inflamatória sistêmica. Se não prevenidas, tais infecções podem provocar complicações e evolução para outros DE, como risco de choque (NANDA International, 2018).

Diante da imunidade comprometida e da associação com comorbidades, o paciente com a COVID-19 encontra-se mais propenso a desenvolver sepse e choque séptico. Acerca disso, estudo realizado com base nos dados de Wuhan, China, identificou que 5\% dos pacientes hospitalizados apresentou essas complicações e neste grupo a taxa de mortalidade foi de 50\% nos pacientes que estavam em Unidade de Terapia Intensiva (UTI) (Cascella; Rajnik; Cuomo; Dulebohn; Di Napoli, 2020). Portanto, os cuidados de enfermagem devem seguir as diretrizes para sepse existentes e têm como alvo a prevenção e a redução do mal prognóstico.

O trato respiratório é um dos locais afetados, causando problemas na ventilação e troca de gases e posteriormente leva a possíveis complicações cardiovasculares. Isso se desencadeia pois na superfície das células epiteliais do pulmão e coração, há a presença da enzima conversora da angiotensina 2 (ECA2), que realiza a mediação da entrada do vírus nas células, que possibilita a replicação viral e consequente resposta inflamatória (Rizzo; Sega; Fortini; Marracino; Rapezzi; Ferrari, 2020).

Os diagnósticos de enfermagem que abordaram a respiração, estão ligados diretamente a esta resposta, corroborando o que foi evidenciado (expectoração, tosse, dispneia/falta de ar, diminuição do $\mathrm{SO}_{2}$ e $\mathrm{PaO}_{2}$, consolidação e opacidade em vidro fosco), tendo como foco causal a produção excessiva de exsudato/muco e lesão alveolar. A nível pulmonar, esta resposta resulta na inflamação nos pulmões, que causa necrose, infiltração e hiperplasia, e acarreta também danos severos das células epiteliais dos alvéolos, dos septos alveolares e infiltração do espaço alveolar (Li, et al, 2020).

Baseados nos problemas evidenciados, todos os resultados e intervenções sugeridos estão voltados para atingir a melhora da permeabilidade das vias aéreas, ventilação e troca de gases (Moorhead; Johnson; Maas; Swanson, 2016; Bulechek; Butcher; Dochterman, 2016; Johnson; Moorhead; Bulechek; Butcher; Maas; Swanson, 2012). Portanto, devem ser implementadas medidas que promovam a desobstrução das vias aéreas, mobilização e eliminação das secreções brônquicas e alveolares, administração de oxigenação suplementar, cuidados com a ventilação mecânica invasiva e não-invasiva e as vias 
aéreas artificiais, cuidados com dreno de tórax, controle e combate a infecção, redução da resposta inflamatória e suas complicações, bem como a monitorização da respiração, de exames laboratoriais e imagem (Joseph, 2020; Jin, et all, 2020).

Os danos causados pela COVID-19 no sistema respiratório, levam ao dano pulmonar, hipoxemia e consequente estresse cardiovascular. Pacientes com comorbidades cardiovasculares são mais propensos a doenças virais e, portanto, constituem um grupo de alto risco e pior prognóstico, com aumento de 5 a 10 vezes na mortalidade (Bonow; Fonarow; O'gara; Yancy, 2020).

Ademais, biomarcadores de lesão cardíaca encontrados no estudo e anormalidades eletrocardiográficas se correlacionam com marcadores inflamatórios elevados. A infecção por SARS-CoV parece diminuir os receptores ECA2, o que pode contribuir para a disfunção ventricular esquerda (Joseph, 2020). Estudo que realizou análise prévia de corações humanos em pacientes que morreram com COVID-19 demonstrou que 35\% corações abrigavam vírus no miocárdio. Não se sabe se o dano cardíaco observado é devido a lesão viral ou devido a uma resposta inflamatória que afeta o miocárdio e estruturas como o pericárdio e sistema de condução (Oudit, et al, 2020).

Dentre as complicações cardiovasculares, destacam-se a insuficiência cardíaca, miocardite, pericardite, vasculite, e arritmias cardíacas (Inciardi, Et Al, 2020; Guo, et al, 2020). Diante de tais complicações cardiovasculares do paciente com COVID-19, podemos identificar no estudo fatores de risco como hipertensão, hipoxemia, PCR elevado, alteração na frequência cardíaca e no ritmo cardíaco, bem como a presença de alterações cardiovasculares, que gerou o diagnóstico de enfermagem com foco no risco de perfusão tissular cardíaca diminuída e risco de débito cardíaco diminuído (NANDA International, 2018).

Algumas intervenções de enfermagem são essenciais para prevenção de complicações cardiovasculares, como oxigenoterapia, monitorização cardíaca, respiratória, sinais vitais, e regulação hemodinâmica (Bulechek; Butcher; Dochterman, 2016). A COVID-19 frequentemente leva a lesão cardíaca aguda, insuficiência cardíaca e arritmias, os quais levam a piores prognósticos. Isso destaca a necessidade de um monitoramento cardíaco precoce e detalhado, especialmente naqueles criticamente doentes (Kunal; Gupta; Sharma; Pathak; Mittal; Tarke, 2020).

Destarte, a vasculite pode levar ao estado protrombótico, onde a elevação deste marcador indireto da geração de trombina leva a ativação da hemostasia e aumento da frequência de embolia, que piora o estado hipoxêmico, aumentando a derivação nessas células altamente hipoxêmicas nos pacientes com SDRA. Isso, combinado com a inflamação sistêmica ou tempestade de citocinas, pode piorar a lesão cardíaca, levar a uma insuficiência cardíaca e ao mau prognóstico. Ressalta-se que os níveis elevados de dímero D representam um marcador de gravidade em casos de COVID-19 (Zhou, Et Al, 2020; Tang; Li; Wang; Sun, 2020).

Pacientes com evidência clínica de vasculite ou indicadores laboratoriais de progressiva inflamação, como aumento dos níveis de IL-6 e/ou dímero D, foram observados na maioria dos estudos desta revisão. Esses e os fatores de risco admissão na UTI, mobilidade prejudicada, idade > 60 anos, comorbidade médica significativa, corroboraram para proposição do diagnóstico de enfermagem risco de tromboembolismo venoso (NANDA International, 2018). Como intervenção de enfermagem é importante destacar a identificação precoce dos pacientes em risco de tromboembolismo, controle de terapia trombolítica, controle na administração de medicamentos trombolíticos, conforme a prescrição médica, e protocolo institucional, cuidados circulatórios como a insuficiência venosa e cuidados na embolia periférica (Bulechek; Butcher; Dochterman, 2016).

Outro achado relevante do estudo foi a presença de problemas gastrointestinais, com destaque para a diarreia, náusea e vômito. Semelhante ao que ocorre nos pulmões e coração, há também uma grande quantidade de ECA2 nas células epiteliais na mucosa do esôfago e intestino. Com isso, os resultados e intervenções sugeridos procuram controlar a infecção, diminuindo a irritabilidade da mucosa intestinal, como também promover o alívio e monitoração dos sintomas, cuidados com os riscos presentes, particularmente o desequilíbrio hidroeletrolítico (Gao; Chen; Fang, 2020).

Os diagnósticos, resultados e intervenções de enfermagem apresentados foram construídos baseados em informações gerais dos artigos apresentados, sendo uma possibilidade de sugestão de problemas e sua resolução. Reforçamos que devem ser 
implementados em um contexto individualizado, bem como com a capacidade de intervenção de cada instituição. Espera-se com a sugestão dos títulos, os profissionais da enfermagem possam ter um direcionamento para facilitar e potencializar as suas ações.

Por meio da síntese dos resultados de estudos que abordaram as características clínicas da COVID-19 foi possível identificar evidências para elaboração de uma proposta de processo de enfermagem, baseado no sistema de classificação da NANDA-I, NOC e NIC. O estudo contribui para implementação da prática de enfermagem por meio do uso de taxonomias padronizadas que permitem a melhoria da qualidade e eficiência da assistência aos pacientes com a COVID-19.

\section{Limitações do estudo}

Como limitação, ressalta-se a dificuldade de comparação dos resultados com outros estudos devido a ausência de investigações sobre a COVID-19 na área de enfermagem até o momento. Presume-se que isto se deu em razão do curto período de tempo transcorrido entre o início da pandemia e a realização deste estudo.

\section{Conclusão}

As características clínicas identificadas nos pacientes com a COVID-19 permitiram a proposição de diagnósticos, resultados e intervenções de enfermagem por meio dos sistemas de classificação NANDA-I, NOC e NIC. Tais diagnósticos tiveram como foco na temperatura corporal, infecção, oxigenação/respiração, cardiovascular e função gastrointestinal. Os resultados foram direcionados para avaliar a progressão dos sinais e sintomas encontrados e as intervenções tiveram como foco o combate às causas dos problemas e o monitoramento do quadro clínico dos infectados.

Diante da relevância da prática da enfermagem apoiada no raciocínio crítico e em uma linguagem unificada, sugere-se a realização de estudos com foco na avaliação individualizada do paciente. Dessa forma, permite-se a proposição de outros diagnósticos, resultados e intervenções que ampliem a prática baseada em evidências para o enfrentamento da epidemia pelo novo coronavírus.

Diante das limitações encontradas para realização do estudo, em detrimento da Sars-cov-2 ser uma doença nova, inferese como sugestões para trabalhos futuros, pesquisas clínicas que busquem uma maior compreensão do mecanismo fisiopatológico do vírus nos sistemas corporais, assim como ensaios clínicos randomizados com o objetivo de avaliar e comparar a eficácia e segurança das intervenções para manejo do paciente acometido pela covid.

\section{Referências}

Alfaro-Lefevre, R. (2014). Aplicação do Processo de enfermagem: um guia passo a passo. (8a ed.), Artmed

Arksey, H., \& O’Malley L. (2005). Scoping studies: towards a methodological framework. Int J Soc Res Methodol. 8, 19-32. 10.1080/1364557032000119616

Bonow, R. O., Fonarow, G. C., O'Gara, P. T., \& Yancy, C. W. (2020). Association of Coronavirus Disease 2019 (COVID-19) With Myocardial Injury and Mortality. JAMA Cardiol. 10.1001/jamacardio.2020.1105.

Bulechek, G. M., Butcher, H. K., \& Dochterman, J. M. C. (2016). Classificação das intervenções de enfermagem (NIC). Tradução Denise Costa Rodrigues. 6.ed. Rio de Janeiro: Elsevier; 2016.

Cai, Q., Huang, D., Ou, P., et al. (2020). COVID-19 in a designated infectious diseases hospital outside Hubei Province, China. Allergy. 1-11. 10.1111/all.14309.

Cao, J., Tu, W. J., Cheng, W., Lei, Y., Liu, Y. K., \& Hu, X. (2020). Clinical Features and Short-term Outcomes of 102 Patients with Coronavirus Disease 2019 in Wuhan, China. Clin Infec Dis. 10.1093/cid/ciaa243

Cao, Y., Liu, X., Xiong, L., \& Cai, K. (2020). Imaging and clinical features of patients with 2019 novel coronavirus SARS-CoV-2: A systematic review and meta-analysis. J Med Virol. 1-11. 10.1002/jmv.25822.

Cascella, M., Rajnik, M., Cuomo, A., Dulebohn, S. C., \& Di Napoli, R. (2020). Features, Evaluation and Treatment Coronavirus (COVID-19). StatPearls [Internet]. https://www.ncbi.nlm.nih.gov/books/NBK554776.

Chen, G., Wu, D., Guo, W., Cao, Y., Huang, D., \& Wang, H. (2020) Clinical and immunologic features in severe and moderate Coronavirus Disease 2019. J Clin Invest. 130(5), 2620-2629. 10.1172/JCI137244. 
Chen, N., Zhou, M., Dong, X., Qu, J., Gong, F., \& Han, Y. (2020). Epidemiological and clinical characteristics of 99 cases of 2019 novel coronavirus pneumonia in Wuhan, China: a descriptive study. The Lancet. 395(10223), 507-513. 10.1016/S0140-6736(20)30211-7.

Chen, T., Wu, D., Chen, H., Yan, W., Yang, D., \& Chen, G. (2020). Clinical characteristics of 113 deceased patients with coronavirus disease 2019: retrospective study. BMJ. 368, m1091. 10.1136/bmj.m1091.

Deng, Y., Liu, W., Liu, K., Fang, Y. Y., Shang, J., Zhou, L. (2020). Clinical characteristics of fatal and recovered cases of coronavirus disease 2019 (COVID19) in Wuhan, China: a retrospective study. Chin Med J. 10.1097/CM9.0000000000000824.

Du, R. H., Liu, L. M., Yi, W., Wang, W., Guan, L. L., \& Yuan, M. L. (2020). Hospitalization and Critical Care of 109 Decedents with COVID-19 Pneumonia in Wuhan, China. Ann Am Thorac Soc. 10.1513/AnnalsATS.202003-225OC.

ECDC. (2020). European Center for disease prevention and control. Coronavirus disease 2019 (COVID-19) in the EU/EEA and the UK - eighth update. https://www.ecdc.europa.eu/sites/default/files/documents/covid-19-rapid-risk-assessment-coronavirus-disease-2019-eighth-update-8-april-2020.pdf.

El-Radhi, A. S. (2019). Pathogenesis of Fever. In: El-Radhi A. (eds) Clinical Manual of Fever in Children. Springer. 10.1007/978-3-319-92336-9_3.

Gao, Q. Y., Chen, Y. X., \& Fang, J. Y. (2020). 2019 Novel coronavirus infection and gastrointestinal tract. J Dig Dis. 21, 125-126. 10.1111/1751-2980.12851.

Garg, S., Kim, L., Whitaker, M., O'Halloran, A., Cummings, C., \& Holstein, R. (2020). Hospitalization Rates and Characteristics of Patients Hospitalized with Laboratory-Confirmed Coronavirus Disease 2019 - COVID-NET, 14 States, 1-30, 2020. Weekly. 69(15), 458-464. https://www.cdc.gov/mm wr/volumes/69/wr/mm6915e3.htm.

Guan, W. J., Ni, Z. Y., Hu, Y., Liang, W. L., Ou, C. Q., \& He, J. X. (2020). Clinical Characteristics of Coronavirus Disease 2019 in China. N Engl J Med. 382 , 1708-1720. 10.1056/NEJMoa2002032.

Guo, T., Fan, Y., Chen, M., Wu, X., Zhang, L., \& He, T. (2020). Cardiovascular Implications of Fatal Outcomes of Patients With Coronavirus Disease 2019 (COVID-19). JAMA Cardiol. 10.1001/jamacardio.2020.1017.

Herdman, T. H., \& Kamitsuru, S. (2018). Diagnóstico de enfermagem da NANDA: definições e classificação 2018-2020 / NANDA International (R. M. Garcez, Trad.). Porto Alegre: Artmed.

Inciardi, R. M., Lupi, L., Zaccone, G., Italia, L., Raffo, M., \& Tomasoni, D. (2020). Cardiac Involvement in a Patient With Coronavirus Disease 2019 (COVID19). JAMA Cardiol. 10.1001/jamacardio.2020.1096.

Jin, Y. H., Cai, L., Cheng, Z. S., Cheng, H., Deng, T., \& Fan, Y. P. (2020). A rapid advice guideline for the diagnosis and treatment of 2019 novel coronavirus (2019-nCoV) infected pneumonia (standard version). Mil Med Res. 7(1), 4. 10.1186/s40779-020-0233-6.

Jonhson, M., Moorhead, S., Bulechek, G., Butcher, H., Maas, M., \& Swanson, E. (2012). Ligações NANDA-NOC-NIC: condições clínicas: suporte ao raciocínio e assistência de qualidade. (R. M. Garcez, \& S. I. Oliveira, Trad.) (3a ed). Elsevier.

Joseph, T. (2020). International Pulmonologist's Consensus on COVID-19. (2a ed.), https://www.saudedafamilia.org/coronavirus/artig os/international_pulmonologists_consensus.pdf.

Kunal, S.; Gupta, K.; Sharma, S. M.; Pathak, V.; Mittal, S., \& Tarke, C. (2020). Cardiovascular system and COVID-19: perspectives from a developing country . Monaldi Arch Chest Dis. 90(2). 10.4081/monaldi.2020.1305.

Li, G., Fan, Y., Lai, Y., Han, T., Li, Z., \& Zhou, P. (2020). Coronavirus infections and immune responses. J Med Virol. 92, 424-432. 10.1002/jmv.25685.

Liu, K., Fang, Y. Y., Deng, Y., Liu, W., Wang, M. F., \& Ma, J. P. (2020). Clinical characteristics of novel coronavirus cases in tertiary hospitals in Hubei Province. Chin Med J. 133(9), 1025-1031. 10.1097/CM9.0000000000000744.

Mo, P., Xing, Y., Xiao, Y., Deng, L., Zhao, Q., \& Wang, H. (2020). Clinical characteristics of refractory COVID-19 pneumonia in Wuhan, China. Clin Infec Dis. $10.1093 / \mathrm{cid} / \mathrm{ciaa} 270$.

Moorhead, S., Johnson, M., Maas, M., \& Swanson, E. (2016). Classificação dos Resultados de Enfermagem (NOC) (A. Fernandes, Trad.) (5a ed.). Elsevier.

Oliveira, S. P., Silva, L. C. R., Silva, P. M.A., \& Chianca, T. C. M. (2016). Physical methods for the treatment of fever in critically ill patients: a randomized controlled trial. Rev. esc. enferm. USP. 50(5), 823-830. 10.1590/s0080-623420160000600016.

Oudit, G. Y., Kassiri, Z., Jiang, C., Liu, P. P., Poutanen, S. M., \& Penninger, J. M. (2009). SARS coronavirus modulation of myocardial ACE2 expression and inflammation in patients with SARS. Eur J Clin Invest. 39, 618-25. 10.1111/j.1365-2362.2009.02153.x.

Pereira, A. S. et al (2018). Metodologia da pesquisa cientifica. UFSM. https://www.ufsm.br/app/uploads/sites/358/2019/02/Metodologia-da-PesquisaCientifica_final.pdf

Rizzo, P., Sega, F. V. D., Fortini, F., Marracino, L., Rapezzi, C., \& Ferrari, R. (2020). COVID-19 in the heart and the lungs: could we "Notch" the inflammatory storm? Basic Res Cardiol. 115(3), 31. 10.1007/s00395-020-0791-5.

Stetler, C.B., Brunell, M., Giuliano, K. K., Morsi, D., Prince, L., \& Newell-Stokes, V. (1998). Evidence-based practice and the role of nursing leadership. J Nurs Adm. 28(7-8), 45-53. 10.1097/00005110-199807000-00011.

Sun, P., Qie, S., Liu, Z., Ren, J., Li, K., \& Xi, J. (2020). Clinical characteristics of hospitalized patients with SARS-CoV-2 infection: A single arm meta-analysis. J Med Virol. 92, 612-617. 10.1002/jmv.25735. 
Research, Society and Development, v. 10, n. 2, e53810212741, 2021

(CC BY 4.0) | ISSN 2525-3409 | DOI: http://dx.doi.org/10.33448/rsd-v10i2.12741

Tang, N., Li, D., Wang, X., \& Sun, Z. (2020). Abnormal coagulation parameters are associated with poor prognosis in patients with novel coronavirus pneumonia J Thromb Haemost. 18(4), 844-847. 10.1111/jth.14768.

Tricco, A. C., Lillie, E., Zarin, W., \& O'Brien, K. K. (2018), Colquhoun H, Levac D, et al. PRISMA extension for scoping reviews (PRISMA-ScR): checklist and explanation. Ann Intern Med. 169(7), 467-473. 10.7326/M18-0850

Wan, S., Xiang, Y., Fang, W., Zheng, Y., Li, B., \& Hu, Y. (2020). Clinical features and treatment of COVID-19 patients in northeast Chongqing. J Med Virol. 10.1002/jmv.25783.

Wang, D., Hu, B., Hu, C., Zhu, F., Liu, X., \& Zhang, J. (2020). Clinical Characteristics of 138 Hospitalized Patients With 2019 Novel Coronavirus-Infected Pneumonia in Wuhan, China. JAMA. 323(11), 1061-1069. doi:10.1001/jama.2020.1585.

Wang, F., Nie, J., Wang, H., Zhao, Q., Xiong, Y., \& Deng, L. (2020). Characteristics of peripheral lymphocyte subset alteration in COVID-19 pneumonia. $J$ Infect Dis. 221(11), 1762-1769. 10.1093/infdis/jiaa150.

Wang, Z., Yang, B., Li, Q., Wen, L., \& Zhang, R. (2020) Clinical Features of 69 Cases with Coronavirus Disease 2019 in Wuhan, China. Clin Infec Dis. $10.1093 / \mathrm{cid} / \mathrm{ciaa} 272$.

Wenham, C., Smith, J., \& Morgan, R. (2020). COVID-19: the gendered impacts of the outbreak. Online. The Lancet. Z395-10227, 846-848. 10.1016/S01406736(20)30526-2.

World Health Organization. (2020). Coronavirus disease (COVID-19) situation Report -119 WHO. https://www.who.int/docs/defaultsource/coronaviruse/situation-reports/20200518-covid-19-sitrep-119.pdf?sfvrsn=4bd9de25_4.

World Health Organization. (2020). State of the World's Nursing Report - 2020. WHO [Internet]. https://www.who.int/publications-detail/nursing-report-2020.

Yang, J., Zheng, Y., Gou, X., Pu, K., Chen, Z., \& Guo, Q. (2020). Prevalence of comorbidities and its effects in patients infected with SARS-CoV-2: a systematic review and meta-analysis. Int J Infect Dis. 94, 91-95. 10.1016/j.ijid.2020.03.017.

Yang, W., Cao, Q., Qin, L., Wang, X., Cheng, Z., \& Pan A. (2020). Clinical characteristics and imaging manifestations of the 2019 novel coronavirus disease (COVID-19): A multi-center study in Wenzhou city, Zhejiang, China. J Infect. 80(4), 388-393. 10.1016/j.jinf.2020.02.016.

Yang, X., Yu, Y., Xu, J., Shu, H., Xia, J., \& Liu, H. (2020). Clinical course and outcomes of critically ill patients with SARS-CoV-2 pneumonia in Wuhan, China: a single-centered, retrospective, observational study. Lancet Respir Med. 8, 475-81. 10.1016/S2213-2600(20)30079-5.

Zhou, F., Yu, T., Du, R., Fan, G., Liu, Y., \& Liu, Z. (2020). Clinical course and risk factors for mortality of adult inpatients with COVID-19 in Wuhan, China: a retrospective cohort study. Lancet. 11(20), 30566-3. 10.1016/S0140- 67 